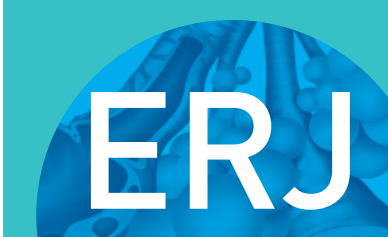

open research



\section{Influence of age, sex and respiratory viruses on the rates of emergency department visits and hospitalisations with respiratory tract infections, asthma and COPD}

\author{
Imran Satia (10 1,2,3,4, Adil Adatia (1) ${ }^{1,2,4}$, Ruth P. Cusack ${ }^{1}$, Justina M. Greene ${ }^{1}$, \\ Paul M. O'Byrne (1) ${ }^{1,2}$, Kieran J. Killian ${ }^{1}$ and Neil Johnston ${ }^{2}$
}

Affiliations: ${ }^{1}$ Dept of Medicine, McMaster University, Hamilton, Canada. ${ }^{2}$ Firestone Institute for Respiratory Health, St Joseph's Healthcare, Hamilton, Canada. ${ }^{3}$ Division of Infection, Immunity and Respiratory Medicine, and Manchester Academic Health Science Centre, University of Manchester, Manchester, UK. ${ }^{4}$ These authors contributed equally.

Correspondence: Imran Satia, McMaster University, Dept of Medicine, Division of Respirology, 1200 Main Street W, Hamilton, ON, L8N 3ZS, Canada. E-mail: satiaiamcmaster.ca

\section{ABSTRACT}

Background: The importance of age, sex and respiratory virus prevalence in emergency department (ED) visits and hospitalisations for respiratory tract infections (RTIs), asthma and COPD in a whole population over time is not well established.

Methods: This study retrospectively analysed data for daily ED visits and hospitalisations from 2003 to 2013 in Ontario, Canada and the daily number of virus positive tests. Daily numbers of ED visits and hospitalisations with RTIs, asthma and COPD listed as a primary diagnosis were collected from the Canadian Institute for Health Information. Virus data were obtained from the Respiratory Virus Detection Surveillance System. Multiple linear regression was used to assess the association of individual viruses with the daily rates.

Results: There were 4365578 ED visits and 321719 (7.4\%) admissions for RTIs, 817141 ED visits and 260665 (31.9\%) admissions for COPD and 649666 ED visits and 68626 (10.6\%) admissions for asthma. Respiratory syncytial virus and influenza A were associated with male ED visits, whereas human rhinovirus was associated with female ED visits for RTIs in preschool children. $19.2 \%$ of males, but only $7.2 \%$ of females were admitted. The correlation between the prevalence of each virus and ED visits and hospitalisations for asthma was weak, irrespective of age group and sex. Influenza A was most strongly associated with COPD ED visits and hospitalisations in males and females.

Conclusions: There are significant age and sex differences in the contribution of respiratory viruses to the number of ED visits and hospitalisations for RTIs, asthma and COPD.

@ERSpublications

There are important age- and sex-related differences in the contribution of respiratory viruses to the number of ED visits and hospitalisations for respiratory tract infections, asthma and COPD https://bit.ly/39hrhIW

Cite this article as: Satia I, Adatia A, Cusack RP, et al. Influence of age, sex and respiratory viruses on the rates of emergency department visits and hospitalisations with respiratory tract infections, asthma and COPD. ERJ Open Res 2021; 7: 00053-2021 [https://doi.org/10.1183/23120541.000532021].
Received: 25 Jan 2021 | Accepted: 18 March 2021

Copyright $\odot$ The authors 2021. This version is distributed under the terms of the Creative Commons Attribution NonCommercial Licence 4.0. For commercial reproduction rights and permissions contact permissions@ersnet.org 


\section{Introduction}

Viral infections are predominantly manifested in diseases of the respiratory system and present to hospitals as respiratory tract infections (RTI) or exacerbations of a pre-existing lung disease such as COPD [1] or asthma [2,3]. At an individual level, the contribution of viruses to clinical disease was investigated between 1946 and 1989 at the Medical Research Council Common Cold Unit (Salisbury, UK) [4, 5]. The inoculation of viral infections led to the recognition of the eight common respiratory viruses. These inoculation techniques continue to the present day [6-9]. This provides unique information about symptoms, cytokine responses and transmission. Today, with the introduction of PCR techniques, the prevalence of these viruses can be monitored throughout the year at a population level.

The Canadian Respiratory Virus Detection Surveillance System [10] reports the percentage of positive tests for eight viruses every week: influenza $A$ and $B$, respiratory syncytial virus (RSV), parainfluenza and adenovirus, human metapneumovirus (hMPV), human rhinovirus (hRV) and coronavirus. In addition, the Canadian Institute for Health Information collects the daily numbers of visits to the emergency department (ED) and the numbers hospitalised for RTI, asthma and COPD. Using these combined datasets, respiratory viruses accounted for $66.7 \%$ of ED visits and $74.4 \%$ of hospitalisations for RTI, $52.5 \%$ of visits and $48.2 \%$ of hospitalisations for COPD, and only $13.3 \%$ of visits and $10.4 \%$ of hospitalisations for asthma [11]. Influenza A and RSV were the dominant viruses explaining the variability in ED visits and hospitalisation for RTIs, while influenza A, hRV and RSV were the dominant viruses for COPD. For asthma, only hRV provided any meaningful contribution to the variability in daily rates in ED visits and hospitalisations [11].

The influence of age and sex on ED visits and hospitalisations and whether specific viruses influence these outcomes differently based on age and sex is potentially important. The young and elderly are prone to developing RTIs and have a higher mortality rate [12-15]. Males aged $<15$ years are more likely to be hospitalised for an exacerbation of asthma or RTI [16]. In contrast, female COPD patients have been associated with more severe exacerbations $[17,18]$ and a faster rising mortality rate in the United States (USA) compared to males between 1980 and 2000 [19]. Between 2000 and 2014, the age-adjusted COPD-related mortality in the USA decreased by $22.5 \%$ in males, but only $3.8 \%$ in females [20].

Many previous studies investigating viruses in RTIs, asthma and COPD have used cohort studies with a relatively small number of highly selected participants. However, within a whole population where there is universal healthcare, and the reasons for ED visits and hospitalisations, along with individual daily virus rates, are being collected, a much broader perspective can be explored.

The objective of this study was first to investigate the effect of age and sex on ED visits and hospitalisations from RTIs, asthma and COPD over 11 years in Ontario, Canada. Second, we sought to investigate the association of individual respiratory viruses within each age and sex category.

\section{Materials and methods}

Data acquisition

The Respiratory Virus Detection Surveillance System obtains the number of daily tests performed and the number of tests positive for respiratory viruses using multiplex PCR from laboratories across Canada. In the province of Ontario, data are collected from 11 public health laboratories and six hospital laboratories and are reported weekly. Data for influenza A and B, RSV, parainfluenza and adenovirus were available for 2003-2013, and hMPV, hRV and coronavirus, which were added to the PCR panel in 2010, were available from 2010 to 2013.

The number of ED visits and hospitalisations with RTI (International Classification of Diseases (ICD)-10 J00-J06, J09-J18, J20-22), asthma (J45), and COPD ( J40-44, J47) listed as a primary diagnosis (diagnosis most responsible for length of stay for hospitalisation data and first diagnostic field for ED data) each day from 2003 to 2013 were obtained from the Canadian Institute for Health Information. Population data for the province of Ontario were obtained from Statistics Canada. Asthma prevalence data were collected from The Ontario Asthma Surveillance Information System and Statistics Canada. The Hamilton Integrated Research Ethics Board waived the requirement for ethics approval, as only aggregate data were obtained.

\section{Virus detection}

The nasopharyngeal swabs were performed on patients presenting to community healthcare practitioners in Ontario, Canada. Specimens were forwarded to the nearest public health laboratory, and viruses were identified by multiplex PCR. These were collected in 17 hospital and public health laboratories across Ontario. The number of tests positive for each virus was published on Saturday every week and expressed as a percentage of the total number of tests performed. The percentage of positive tests for each virus derived for each week was assumed to apply equally to every day of the previous week. 


\section{Statistical analysis}

The daily rates for ED visits and hospitalisations with RTI, asthma and COPD were calculated by dividing the number of events that day by the population of the province at that time and then averaging across the entire study period to obtain the mean event rate in males and females for each of five age categories ( $<5$ years, $5-<15$ years, $15-<50$ years, $50-70$ years and $>70$ years). The ratio of ED visits to admissions was calculated by dividing the mean daily rate of admissions by the mean daily rate of ED visits and expressed as a percent. The $95 \%$ confidence intervals for these percentages were determined by bootstrapping using the bias corrected and accelerated percentile method.

Multiple linear regression with the percentage of nasopharyngeal swabs positive for each virus as regressors and an intercept term was performed using log-transformed rates of RTI, asthma and COPD ED visits and hospitalisations for the five age groups in males and females. There were no days on which the number of ED visits or admissions for asthma, COPD or RTIs in Ontario over the study period was zero, so no data were excluded to facilitate log transformation. The coefficients were expressed as standardised $\beta$-values to compare the strength of each individual virus to the variability in RTI, asthma and COPD ED visits and hospitalisations. Standardised $\beta$ is a measure that expresses how many standard deviations of change in the dependent variable result from a 1-SD change in the predictor variable. Standardised $\beta$-values $<0.1$ for viruses were deemed clinically irrelevant and are not shown in the figures. Analyses were performed in TIBCO Statistica (Academic Package v13.2) and MATLAB Statistical Toolbox (Release 2015a).

\section{Results}

\section{Overall daily ED visits, hospitalisation and percentage of positive viruses}

The population of Ontario increased from 12251405 in 2003 to 13606541 in 2013 . There were 4365578 ED visits for RTIs, of which 321719 (7.4\%) were admitted to hospital; 817141 ED visits for COPD, of which 260665 (31.9\%) were admitted; and 649666 ED visits for asthma, of which 68626 (10.6\%) were admitted. The daily number of ED visits and hospitalisations over 11 years was highest for RTI (mean \pm SD $1087 \pm 426.8$ and $80.1 \pm 31.5$, respectively), followed by COPD (203.4 \pm 61.9 and $64.9 \pm 17.3$, respectively) and lowest for asthma (161.7 \pm 52.9 and $17.1 \pm 8.3$, respectively).

The data for the weekly reports of eight viruses was available from September 2010 until September 2013. Overall, there were 159691 tests performed in the whole of Ontario, Canada. The percentages of all eight respiratory viruses are shown in table $1 . \mathrm{hRV}$ had the highest overall positive rate at nearly $10 \%$, followed by influenza A, RSV, parainfluenza, coronavirus, hMPV, influenza B and adenovirus, in decreasing order.

\section{Daily ED visits and hospitalisations by age and sex \\ Respiratory tract infections}

The daily rates of ED visits and hospitalisations for RTIs across all age groups and sex are shown in figure 1a. The rate of ED visits was higher for females for the age groups $<5$ years, $15-<50$ years and $50-70$ years, but was similar in the $5-<15$-year-olds and was higher in males aged $>70$ years. In both sexes, the rate declined with increasing age until $>70$ years, after which there was an increase. The daily rate of hospitalisations for RTIs was higher in males compared to females in the age groups $<5$ years, 50-70 years and $>70$ years, but were similar in the age groups $5-<15$ years and $15-<50$ years. This was most pronounced in the $<5$-years age group in which $19.2 \%$ (95\% CI 18.8-19.7\%) of males, but only $7.2 \%(7.0-7.3 \%)$ of females

\section{TABLE 1 The positive rate of virus detection in Ontario, Canada from 2010 to 2013}

\begin{tabular}{llccccc} 
& \multicolumn{7}{c}{ Positive rate of virus detection \% } \\
\cline { 2 - 6 } & Mean & Median & Lower quartile & Upper quartile & Minimum & Maximum \\
\hline Rhinovirus & 9.96 & 8.75 & 3.79 & 13.47 & 0.62 & 39.46 \\
RSV & 5.30 & 2.20 & 0.88 & 8.40 & 0.00 & 22.97 \\
Influenza A & 4.28 & 1.17 & 0.27 & 3.54 & 0.00 & 32.94 \\
$\begin{array}{l}\text { Parainfluenza } \\
\text { Coronavirus }\end{array}$ & 4.08 & 3.66 & 1.85 & 5.84 & 0.21 & 12.90 \\
hMPV & 2.37 & 1.56 & 0.00 & 3.45 & 0.00 & 12.47 \\
Influenza B & 2.05 & 0.93 & 0.29 & 3.53 & 0.00 & 9.37 \\
Adenovirus & 2.00 & 0.30 & 0.00 & 2.03 & 0.00 & 23.17 \\
& 0.96 & 0.86 & 0.49 & 1.29 & 0.00 & 3.41 \\
\hline $\begin{array}{l}\text { The total number } \\
\text { metapneumovirus. }\end{array}$ &
\end{tabular}



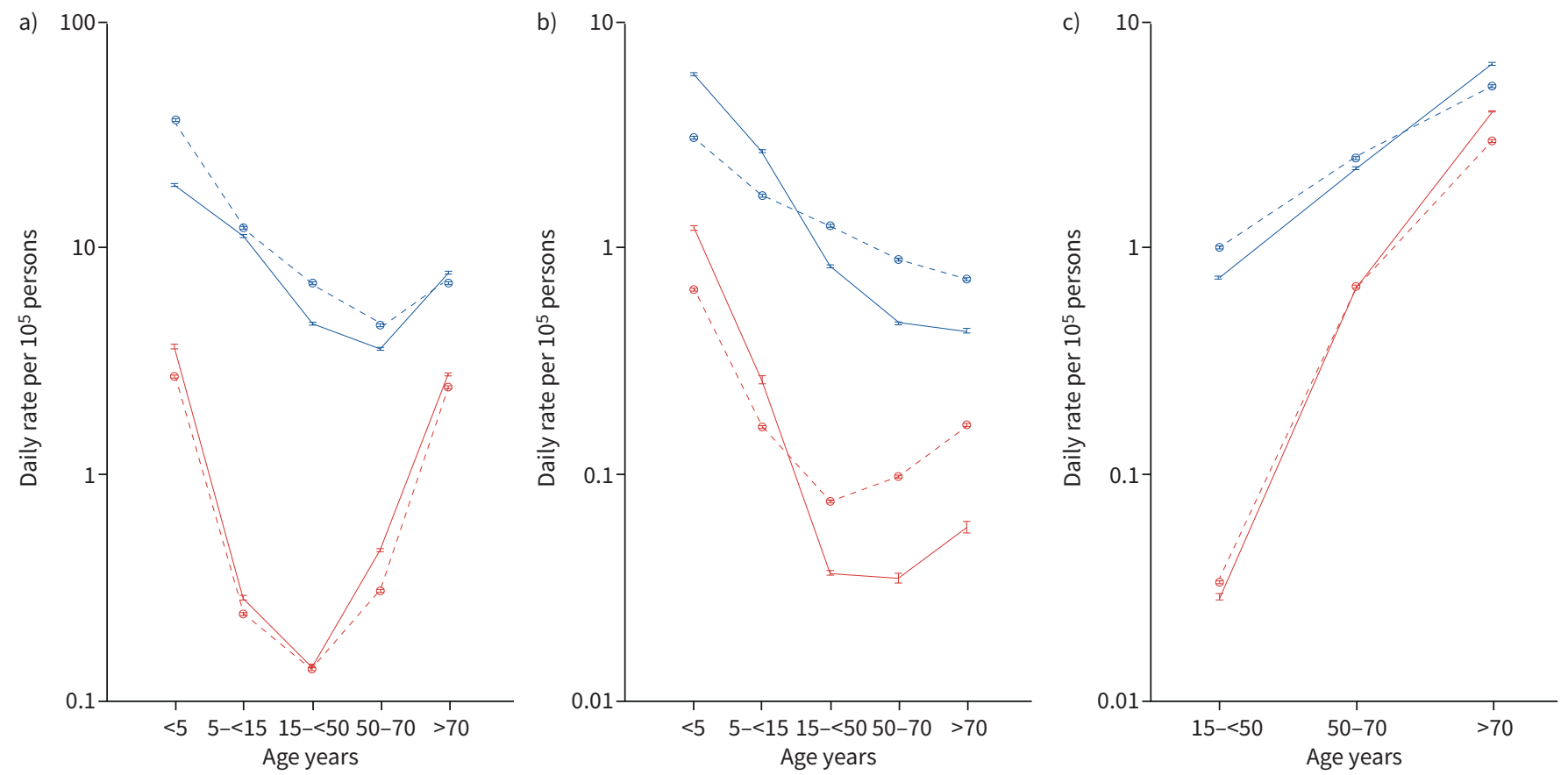

- Male ED visits - - Female ED visits _- Male admissions - - - Female admissions

FIGURE 1 Mean daily rates of emergency department (ED) visits and hospitalisations per $10^{5}$ persons in Ontario, Canada from 2003 to 2013 for a) respiratory tract infections (RTIs), b) asthma and c) COPD. y-axis expressed on a $\log _{10}$ scale and is 10 -fold greater for RTIs. Error bars represent $95 \%$ confidence intervals.

were admitted (table 2). In both sexes, RTI-related hospitalisations declined until the age of 50 years, followed by a sharp increase, with over one-third of those aged $>70$ years being admitted (table 2 ).

\section{Asthma}

The daily rates of ED visits and hospitalisations for asthma across all age groups and sex are shown in figure $1 \mathrm{~b}$. The rate of ED visits was higher for males in the $<5$-years and $5-<15$-years age groups, but was higher in females in the 15-<50-years, 50-70-years and $>70$-years age groups. There was a decline in ED visits for asthma in both sexes, but the rate of decline was greater for males between ages $5-<15$ years and $50-70$ years. The daily rate of hospitalisations for asthma was higher in males in the age groups $<5$ years and $5-<15$ years, but the ratio of ED visits to admissions was similar for the two sexes (see table 2 ). In patients aged $<5$ years, $20.8 \%(20.4-21.2 \%)$ of males and $21.5 \%(21.1-22.1 \%)$ of females were admitted, and in those aged $5-<15$ years, $9.8 \%(9.5-10.1 \%)$ of males and $9.5 \%(9.2-9.8 \%)$ of females were admitted.

TABLE 2 The ratio of the mean daily rate of admissions to the mean daily rate of emergency department visits expressed as a percentage for respiratory tract infection (RTI), asthma and COPD by age group and sex in Ontario, Canada from 2003 to 2013

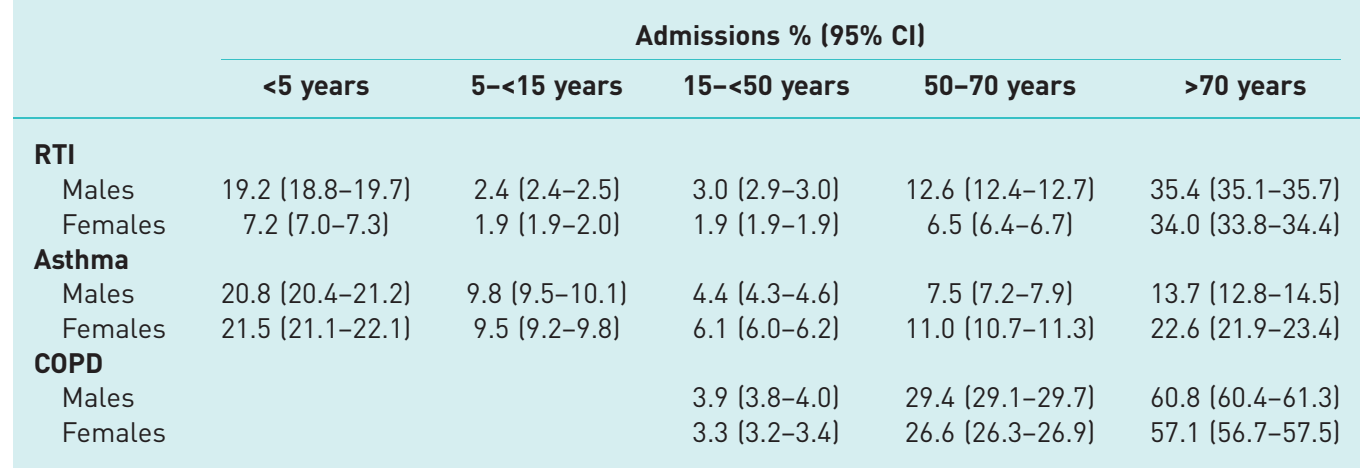


In the age categories from $15-<50$ years to $>70$ years, the daily rate of admissions and the ratio of ED visits to admissions was higher for females. For example, in the $>70$-years group, $22.6 \%(21.9-23.4 \%)$ of females but only $13.7 \%$ (12.8-14.5\%) of males were admitted after an ED visit.

\section{COPD}

The daily rates of ED visits and hospitalisations for COPD across all age groups and sex are shown in figure 1c. The rate of ED visits was higher in females in the $15-<50$-years age category, but the rate of increase by age was higher in males, resulting in a higher daily ED visit rate for males in the $>70$-years age group. The daily rate of hospitalisations increased with age for both sexes and was similar between males and females for between the ages of 15 and 70 years, but was higher in males after the age of 70 years. The ratio of $\mathrm{ED}$ visits to hospitalisations for COPD increased by approximately seven-fold from $15-<50$ years to 50-70 years and two-fold from 50-70 years to $>70$ years such that almost two-thirds of those aged $>70$ years were hospitalised (table 2). Differences in sex in COPD-related hospitalisations were small across all age groups (figure $1 \mathrm{c}$ and table 2).

\section{Association of respiratory viruses with ED visits and hospitalisations by age and sex}

Influenza A, RSV and hRV were selected for representation in all figures, as the remaining viruses (influenza B, adenovirus, coronavirus, hMPV and parainfluenza) were not meaningfully associated with the rates of $\mathrm{ED}$ visits and hospitalisations (standardised $\beta$-values $<0.1$ ).

\section{Respiratory tract infections}

The standardised $\beta$-values representing the association of influenza A, RSV and hRV with RTI ED visits is shown in figure 2a. Influenza A was the most strongly associated with ED visits across all age groups in males and females. Influenza A had a stronger association in males aged $<5$ years, but RSV had a stronger association in females aged $<5$ years. In both sexes between the ages of 5 and 15 years, the strength of association for influenza A was similar to that of RSV. The strength of association of hRV was lower for all age groups in both males and females, but was greater in males compared to females aged $<5$ years.

The standardised $\beta$-values representing the association of influenza A, RSV and hRV with RTI-related hospitalisations are shown in figure $2 \mathrm{~b}$. RSV was most strongly associated with RTI-related hospitalisations in $<5$-year-olds, and then decreased with age. The association of influenza A increased with age and was the most strongly associated virus in the $>70$-years age category. Influenza A was modestly associated in males aged 50-70 years, but not in females. The association with hRV was low for all age groups, with no differences between the sexes.

\section{Asthma}

The standardised $\beta$-values representing the association of influenza A, RSV and hRV with asthma ED visits and hospitalisations are shown in figure $3 \mathrm{a}$ and $\mathrm{b}$, respectively. Rhinovirus was modestly associated with $\mathrm{ED}$ visits, which peaked in the $15-<50$-years age category. Influenza A was not associated until the age of 15 years, after which there was a small increase in standardised $\beta$-values in those aged 50-70 years
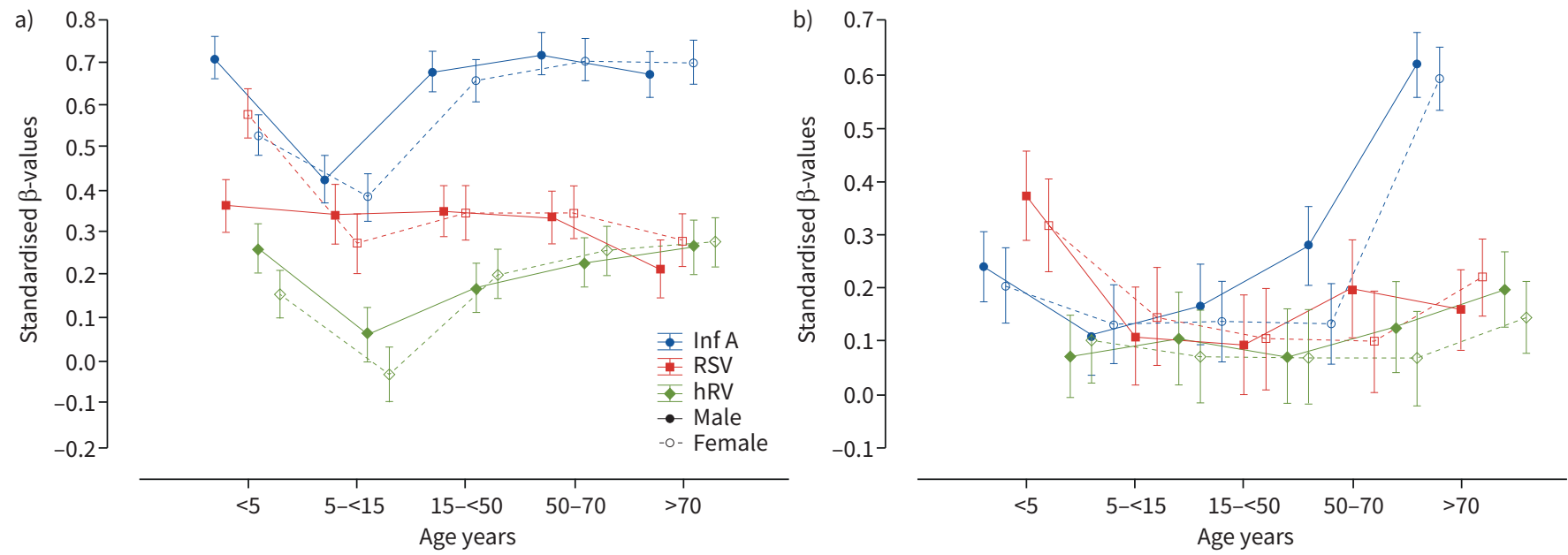

FIGURE 2 Association of influenza A (Inf A), respiratory syncytial virus (RSV) and human rhinovirus (hRV) with respiratory tract infection-related a) emergency department visits and b) hospitalisations in Ontario, Canada for males and females in different age groups from 2010 to 2013. 

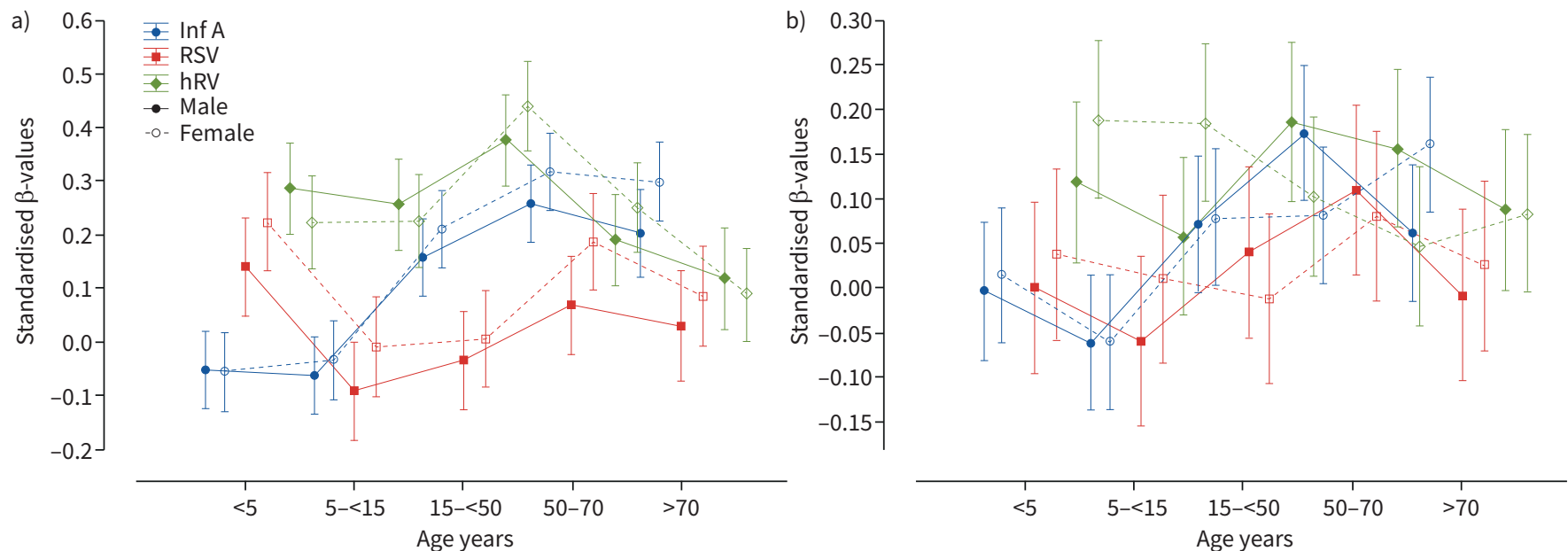

FIGURE 3 Association of influenza A (Inf A), respiratory syncytial virus (RSV) and human rhinovirus (hRV) with asthma-related a) emergency department visits and b) hospitalisations in Ontario, Canada for males and females in different age groups from 2010 to 2013.

and $>70$ years. RSV was weakly associated only in those aged $<5$ years. There were no differences in the association of viruses between the sexes.

The association of all three viruses with asthma hospitalisations was minimal, with no differences between the sexes (figure $3 b$ ).

\section{COPD}

The standardised $\beta$-values representing the association of influenza A, RSV and hRV with COPD-related $\mathrm{ED}$ visits are shown in figure $4 \mathrm{a}$. Influenza $\mathrm{A}$ was the most strongly associated with ED visits for all age groups and was similar in both sexes. The standardised $\beta$-values for hRV were small, and were similar across age groups within each sex, but they were consistently higher for females. The association of RSV was minimal in all age categories and was similar in both males and females.

The standardised $\beta$-values representing the association of influenza A, RSV and hRV to COPD-related hospitalisations is shown in figure $4 \mathrm{~b}$. The association of all viruses was minimal in the $15-<50$-years age category. Influenza A was the most strongly associated virus in the 50-70-years and $>70$-years age groups and this association increased with age. In males and females, the association of influenza A was similar until 70 years, after which it was greater in females. The $\beta$-values for hRV in $50-70$-year-olds in both sexes were small, but in males aged $>70$ years the association became minimal, whereas in females it persisted. RSV was minimally associated in males across all age groups, but this increased with age in females and was thus more strongly associated in the $>70$-years age category.
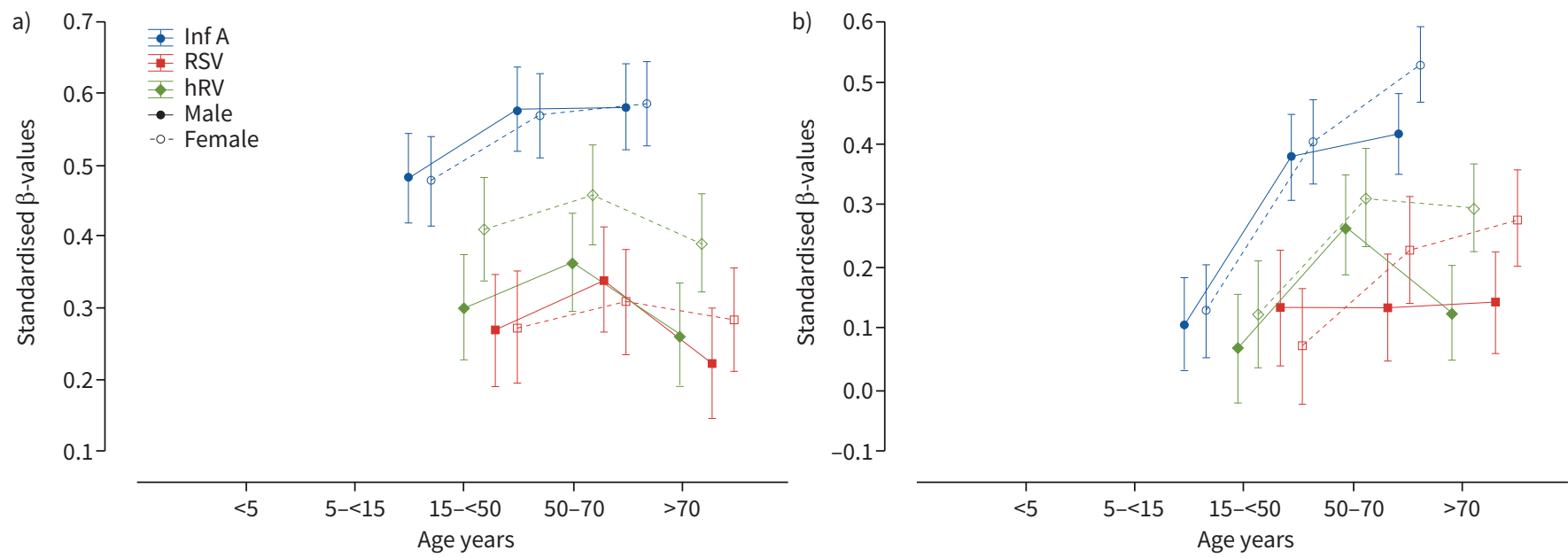

FIGURE 4 Association of influenza A (Inf A), respiratory syncytial virus (RSV) and human rhinovirus (hRV) with COPD-related a) emergency department visits and b) hospitalisations in Ontario, Canada for males and females in different age groups from 2010 to 2013. 


\section{Discussion}

To our knowledge, this is the first study to evaluate age and sex differences in the rates of ED visits and hospitalisations for RTI, asthma and COPD, and concurrently assess the association of respiratory viruses with the observed rates at the population level. The primary findings were 1) the ratio of ED visits to hospitalisation for RTI in males was significantly higher than that of females aged $<5$ years and between 50 and 70 years; 2) the correlation between community respiratory virus prevalence and asthma exacerbations needing ED or inpatient care was weak in all age groups; 3) the correlation between the community prevalence of influenza $\mathrm{A}$ and COPD exacerbations requiring $\mathrm{ED}$ and inpatient care was significantly greater than that of other respiratory viruses; and 4) influenza A and RSV were more strongly associated with RTI ED visits in boys compared to girls aged $<5$ years, whereas hRV was more strongly associated in girls in the same age group.

A higher rate of hospitalisation among male compared to female preschoolers for RTI has been reported [16], although we observed a 2.67 -fold difference compared to the 1.45 -fold difference previously noted. Given that females aged $<5$ years had a higher rate of ED visits for RTI, but were admitted less often, it appears that this trend is due to increased RTI severity in preschool males. At birth, females have more developed lungs based on histological appearance [21], greater surfactant production [22] and have higher forced expiratory flow rates corrected for lung size (termed dysanapsis) [23-25]. These factors may explain the observed differences in this study. Preferential admission of males is an unlikely explanation in Canada, where the public healthcare system bears the entire cost of inpatient care.

Many authors have reported a high rate of respiratory virus positivity in asthmatics during exacerbations, ranging from $50 \%$ to $85 \%$ [26-29], and hRV is the most common virus identified [30]. The asthma epidemic seen in September in many countries has consequently been attributed to an antecedent increase in hRV infection rates [31,32]. Discordance between community virus spread and asthma exacerbations at the population level is topical, given that asthmatics seems to be underrepresented in the current coronavirus disease 2019 (COVID-19) pandemic [33-35]. This study showed a weak temporal correlation between community respiratory virus prevalence (including hRV) and ED visits and hospitalisations for asthma independently of age and sex. We speculate the explanation for this discordance is that those previous studies followed well-selected patients longitudinally, whereas we assessed the relationship between community virus prevalence and asthma exacerbations at the population level. Second, yearly rates of asthma-related $\mathrm{ED}$ visits and hospitalisation are low and decreasing, and hence many milder asthma exacerbations will be managed by primary care physicians and not captured in this dataset. Third, our previous study has demonstrated that $\mathrm{hRV}$ is more prevalent when the prevalence of influenza A and RSV are low. The prevalence of hRV in the community is high when rates of ED visits and hospitalisations are low [11]. Finally, the concomitant role of seasonal allergens must also be considered since allergic sensitisation is associated with worse disease control during hRV-triggered asthma exacerbations [36]. This study was not designed to capture such exposures or allergic status. We found that influenza A was not associated with asthma ED visits and hospitalisations in children aged $<15$ years, but this association was greater in older adults, which corroborates the findings of a previous study on influenza-related asthma exacerbations in Ontario, Canada [37]. Our study expands on this finding by showing that hRV as expected is most strongly associated with asthma morbidity in patients aged $<15$ years, influenza A is weakly associated with asthma exacerbations despite being strongly associated with RTIs in children, and that these differential effects of hRV and influenza A in different age groups is independent of sex.

Our data show that the rate of ED visits and hospitalisations in asthmatics is higher in males in the paediatric population and higher in females in the adult population, which is consistent with the known sex disparity in asthma prevalence [38-40]. The ratio of ED visits to hospitalisations for asthma in adult females was significantly greater compared to adult males. This could possibly be explained by females with asthma demonstrating hypersensitive and hyperresponsive airway sensory nerves, which could result in more severe symptoms, such as cough [41]. This is concordant with other reports [42-44] and probably corresponds to the higher rate of severe asthma in adult females [45-47].

The respiratory virus most commonly associated with COPD exacerbations is hRV [48-51], although others such as influenza A, parainfluenza and RSV are also recognised as important [52-54]. We found that the correlation between community virus prevalence and ED visits and hospitalisations for COPD was strongest for influenza A. In stable COPD patients, a respiratory virus can be detected by PCR in $10-20 \%$ of patients, representing asymptomatic infection, and hRV is also the most commonly identified virus in these patients $[48,50,51]$. In contrast, influenza $\mathrm{A}$ is uncommonly found in COPD patients without symptoms of viral illness or disease exacerbation [48,51], so a high correlation between influenza A prevalence in the community and COPD ED visits and hospitalisations is expected. 
We observed the highest rates of hospitalisation for RTI in young children and older adults, both well-established high-risk groups known to have high rates of RSV and influenza A, respectively [55]. However, pandemic respiratory viruses do not necessarily have a greater effect on young children and older adults. During the 2009-2010 H1N1 influenza pandemic, those aged $>65$ years were significantly less affected compared with nonpandemic influenza seasons [56, 57]. Conversely, children are less severely affected by COVID-19 [58]. Pre-existing immunity has been proposed as the explanation in the case of H1N1 [59], but would not apply to the case of COVID-19 in children. The differential impact of pandemic respiratory viruses in different age groups compared to seasonal viruses requires further study given the substantial public health implications.

Our study has several important limitations. First, the database recording daily rates of ED visits and hospitalisations is not linked to the Respiratory Virus Detection Surveillance System. Consequently, a positive nasopharyngeal swab cannot be linked to a specific ED visit or hospitalisation to establish whether an asthma or COPD exacerbation was due to a viral aetiology. This also prevents assessment of the impact of co-infection with multiple viruses or bacterial infections. Second, we were limited to the age categories available for ED visits and hospitalisations and hence more granular stratification of the data by age was not possible. Third, the number of ED visits and hospitalisations due to each condition are determined using the ICD-10 codes applied by physicians and administrators to each visit, so there is potential for misclassification. A past medical history of COPD or asthma, or the identification of certain viruses during the visit, may have confounding effects on the subsequent ICD-10 coding for a patient presenting with a viral infection. Fourth, the percentage of ED visits resulting in admission was estimated by dividing the number of ED visits by the number of admissions for that time period for each condition, but it is possible for patients to be admitted without attending the ED. Fifth, since we employed population-level databases, we do not have access to confounding factors such as medical comorbidities and medication usage. Sixth, we do not know when the nasopharyngeal swabs were obtained relative to the onset of symptoms, which could impact the test results. Seventh, laboratories choose and validate their own multiplex PCR assays used for virus testing, although each laboratory undergoes audits for quality assurance that are conducted by the Institute for Quality Management in Healthcare. Eight, the virus data were available on a weekly basis, while the rates of ED visits and hospitalisations were on a daily basis. Weekly virus data were assumed to apply equally throughout the week.

\section{Conclusions}

The rate of hospitalisation for RTIs is higher in preschool boys compared to girls. ED visits for RTIs in preschool boys are most strongly associated with influenza A and RSV, but with hRV in girls. ED visits and hospitalisations for asthma were higher in males among children but higher in females among adults. Respiratory viruses are modestly associated with asthma ED visits and hospitalisations, independently of age and sex. ED visits for COPD were higher in males compared to females aged $>70$ years. Influenza A is the respiratory virus most strongly associated with ED visits and hospitalisations for COPD in both males and females.

Author contributions: N. Johnston, K.J. Killian and P.M. O’Byrne conceptualised and designed the study. I. Satia, A. Adatia and K.J. Killian performed data analyses and interpretation. All authors had full access to all the data, and contributed to data analysis and interpretation, and writing of the manuscript.

Conflict of interest: I. Satia reports personal fees from educational talks for general practitioners from GSK and AstraZeneca, grants and personal fees from Merck Canada, a European Respiratory Society Respire 3 Marie Curie Fellowship, and an E.J. Moran Campbell Early Career Award from the Dept of Medicine, McMaster University, outside the submitted work. A. Adatia reports a fellowship award from the Canadian Institute for Health Research/Canadian Allergy, Asthma, and Immunology Foundation/AstraZeneca/AllerGen Emerging Researcher Award in Allergic Asthma outside the submitted work. R.P. Cusack has nothing to disclose. J.M. Greene has nothing to disclose. P.M. O'Byrne reports grants and personal fees from AstraZeneca, personal fees from GSK, grants from Novartis, grants and personal fees from Medimmune, and personal fees from Chiesi, outside the submitted work. K.J. Killian has nothing to disclose. N. Johnston has nothing to disclose.

Support statement: I. Satia is supported by the E.J. Moran Campbell Early Career Award, Department of Medicine, McMaster University. A. Adatia is supported by the Canadian Institute for Health Research/Canadian Allergy, Asthma, and Immunology Foundation/AstraZeneca/AllerGen Emerging Researcher Award in Allergic Asthma. The research reported in this article was funded by an unrestricted grant from AstraZeneca Canada with no input in data collection, analysis, interpretation or writing of the manuscript. Funding information for this article has been deposited with the Crossref Funder Registry.

\section{References}

1 Beckham JD, Cadena A, Lin J, et al. Respiratory viral infections in patients with chronic, obstructive pulmonary disease. J Infect 2005; 50: 322-330. 
2 Busse WW, Lemanske RF Jr, Gern JE. Role of viral respiratory infections in asthma and asthma exacerbations. Lancet 2010; 376: 826-834.

3 Ritchie AI, Farne HA, Singanayagam A, et al. Pathogenesis of viral infection in exacerbations of airway disease. Ann Am Thorac Soc 2015; 12: Suppl. 2, S115-132.

4 Greenhough B. Where species meet and mingle: endemic human-virus relations, embodied communication and more-than-human agency at the Common Cold Unit 1946-90. Cult Geogr 2012; 19: 281-301.

$5 \quad$ Tyrrell D. A view from the common cold unit. Antiviral Res 1992; 18: 105-125.

6 Lambkin-Williams R, Noulin N, Mann A, et al. The human viral challenge model: accelerating the evaluation of respiratory antivirals, vaccines and novel diagnostics. Respir Res 2018; 19: 123.

7 Bush RK, Busse W, Flaherty D, et al. Effects of experimental rhinovirus 16 infection on airways and leukocyte function in normal subjects. J Allergy Clin Immunol 1978; 61: 80-87.

8 Higgins $\mathrm{P}, \mathrm{Al}-\mathrm{Nakib} \mathrm{W}$, Willman J, et al. Interferon-beta ser as prophylaxis against experimental rhinovirus infection in volunteers. J Interferon Res 1986; 6: 153-159.

9 Lambkin-Williams R, Mann A, Gilbert A. Re-inventing the Common Cold Institute for the 21st century. J Hum Virol Retrovirol 2016; 3: 00075.

10 Public Health Agency of Canada. Respiratory Virus Detections in Canada. 2020 Available from: www.canada.ca/ en/public-health/services/surveillance/respiratory-virus-detections-canada.html Date last accessed: 1 March 2020.

11 Satia I, Dua B, Singh N, et al. Tracheobronchomegaly, cough and recurrent chest infection: Mounier-Kuhn syndrome. ERJ Open Res 2020; 6: 00138-2020.

12 van Asten L, van den Wijngaard C, van Pelt W, et al. Mortality attributable to 9 common infections: significan effect of influenza A, respiratory syncytial virus, influenza B, norovirus, and parainfluenza in elderly persons. J Infect Dis 2012; 206: 628-639.

13 Sehgal V, Sethi G, Sachdev H, et al. Predictors of mortality in subjects hospitalized with acute lower respiratory tract infections. Indian Pediatr 1997; 34: 213-219.

14 Troeger C, Forouzanfar M, Rao PC, et al. Estimates of the global, regional, and national morbidity, mortality, and aetiologies of lower respiratory tract infections in 195 countries: a systematic analysis for the Global Burden of Disease Study 2015. Lancet Infect Dis 2017; 17: 1133-1161.

15 Hak E, Rovers M, Kuyvenhoven M, et al. Incidence of GP-diagnosed respiratory tract infections according to age, gender and high-risk co-morbidity: the Second Dutch National Survey of General Practice. Fam Pract 2006; 23 291-294.

16 Jensen-Fangel S, Mohey R, Johnsen SP, et al. Gender differences in hospitalisation rates for respiratory tract infections in Danish youth. Scand J Infect Dis 2004; 36: 31-36.

17 Kilic H, Kokturk N, Sari G, et al. Do females behave differently in COPD exacerbation? Int J Chron Obstruct Pulmon Dis 2015; 10: 823-30.

18 Prescott E, Bjerg AM, Andersen PK, et al. Gender difference in smoking effects on lung function and risk of hospitalization for COPD: results from a Danish longitudinal population study. Eur Respir J 1997; 10: 822-827.

19 Mannino DM, Homa DM, Akinbami LJ, et al. Chronic obstructive pulmonary disease surveillance - United States, 1971-2000. Respir Care 2002; 47: 1184-1199.

$20 \mathrm{Ni} \mathrm{H}, \mathrm{Xu}$ J. COPD-related mortality by sex and race among adults aged 25 and over: United States, 2000-2014. NHCS Data Brief, no 256. Hyattsville, National Center for Health Statistics, 2016. www.cdc.gov/nchs/data/ databriefs/db256.pdf

21 Naeye RL, Freeman RK, Blanc WA. Nutrition, sex, and fetal lung maturation. Pediatr Res 1974; 8: 200-204.

22 Torday J, Nielsen H. The sex difference in fetal lung surfactant production. Exp Lung Res 1987; 12: 1-19.

23 Becklake MR, Kauffmann F. Gender differences in airway behaviour over the human life span. Thorax 1999; 54: $1119-1138$.

24 Mead J. Dysanapsis in normal lungs assessed by the relationship between maximal flow, static recoil, and vital capacity. Am Rev Respir Disease 1980; 121: 339-342.

25 Hibbert M, Lannigan A, Raven J, et al. Gender differences in lung growth. Pediatr Pulmonol 1995; 19: 129-134.

26 Johnston SL, Pattemore PK, Sanderson G, et al. Community study of role of viral infections in exacerbations of asthma in 9-11 year old children. BMJ 1995; 310: 1225-1229.

27 McIntosh K, Ellis EF, Hoffman LS, et al. The association of viral and bacterial respiratory infections with exacerbations of wheezing in young asthmatic children. J Pediatr 1973; 82: 578-590.

28 Minor TE, Dick EC, DeMeo AN, et al. Viruses as precipitants of asthmatic attacks in children. JAMA 1974; 227: 292-298.

29 Henderson J, Hilliard TN, Sherriff A, et al. Hospitalisation for RSV bronchiolitis before 12 months of age and subsequent asthma, atopy and wheeze: a longitudinal birth cohort study. Pediatr Allergy Immunol 2005; 16: 386-392.

30 Zheng X-Y, Xu Y-J, Guan W-J, et al. Regional, age and respiratory-secretion-specific prevalence of respiratory viruses associated with asthma exacerbation: a literature review. Arch Virol 2018; 163: 845-853.

31 Johnston NW, Johnston SL, Norman GR, et al. The September epidemic of asthma hospitalization: school children as disease vectors. J Allergy Clin Immunol 2006; 117: 557-562.

32 Dicpinigaitis P, Satia I, Ferguson N. Falsely accused? Insufficient evidence to conclude that sitagliptin is a cause of chronic cough. Lung 2020; 198: 271-273.

33 Halpin DM, Faner R, Sibila $\mathrm{O}$, et al. Do chronic respiratory diseases or their treatment affect the risk of SARS-CoV-2 infection? Lancet Respir Med 2020; 8: 436-438.

34 Satia I, Adatia A, Yaqoob S, et al. Emergency department visits and hospitalisations for asthma, COPD and respiratory tract infections: what is the role of respiratory viruses, and return to school in September, January and March? ERJ Open Res 2020; 6: 00593-2020.

35 Huang C, Wang Y, Li X, et al. Clinical features of patients infected with 2019 novel coronavirus in Wuhan, China. Lancet 2020; 395: 497-506.

36 Green RM, Custovic A, Sanderson G, et al. Synergism between allergens and viruses and risk of hospital admission with asthma: case-control study. BMJ 2002; 324: 763.

37 Feldman LY, Zhu J, To T. Estimating age-specific influenza-associated asthma morbidity in Ontario, Canada. Respir Med 2019; 155: 104-112. 
pub-eng.pdf

41 Satia I, Tsamandouras N, Holt K, et al. Capsaicin-evoked cough responses in asthmatic patients: evidence for airway neuronal dysfunction. J Allergy Clin Immunol 2017; 139: 771-779.

42 Skobeloff EM, Spivey WH, St Clair SS, et al. The influence of age and sex on asthma admissions. JAMA 1992; 268: 3437-3440.

43 Hyndman S, Williams D, Merrill S, et al. Rates of admission to hospital for asthma. BMJ 1994; 308: 1596-1600.

44 Chen Y, Stewart P, Johansen H, et al. Sex difference in hospitalization due to asthma in relation to age. J Clin Epidemiol 2003; 56: 180-187.

45 ENFUMOSA Study Group. The ENFUMOSA cross-sectional European multicentre study of the clinical phenotype of chronic severe asthma. Eur Respir J 2003; 22: 470-477.

46 Gupta D, Keogh B, Chung KF, et al. Characteristics and outcome for admissions to adult, general critical care units with acute severe asthma: a secondary analysis of the ICNARC Case Mix Programme Database. Crit Care 2004; 8: R112-R121

47 Watson L, Turk F, James P, et al. Factors associated with mortality after an asthma admission: a national United Kingdom database analysis. Respir Med 2007; 101: 1659-1664.

48 Seemungal T, Harper-Owen R, Bhowmik A, et al. Detection of rhinovirus in induced sputum at exacerbation of chronic obstructive pulmonary disease. Eur Respir J 2000; 16: 677-683.

49 Papi A, Bellettato CM, Braccioni F, et al. Infections and airway inflammation in chronic obstructive pulmonary disease severe exacerbations. Am J Respir Crit Care Med 2006; 173: 1114-1121.

50 Rohde G, Wiethege A, Borg I, et al. Respiratory viruses in exacerbations of chronic obstructive pulmonary disease requiring hospitalisation: a case-control study. Thorax 2003; 58: 37-42.

51 McManus TE, Marley A-M, Baxter N, et al. Respiratory viral infection in exacerbations of COPD. Respir Med 2008; 102: 1575-1580

52 Tan WC, Xiang X, Qiu D, et al. Epidemiology of respiratory viruses in patients hospitalized with near-fatal asthma, acute exacerbations of asthma, or chronic obstructive pulmonary disease. Am J Med 2003; 115: $272-277$.

53 Greenberg SB, Allen M, Wilson J, et al. Respiratory viral infections in adults with and without chronic obstructive pulmonary disease. Am J Respir Crit Care Med 2000; 162: 167-173.

54 Falsey AR, Formica MA, Hennessey PA, et al. Detection of respiratory syncytial virus in adults with chronic obstructive pulmonary disease. Am J Respir Crit Care Med 2006; 173: 639-643.

55 Schanzer DL, Saboui M, Lee L, et al. Burden of influenza, respiratory syncytial virus, and other respiratory viruses and the completeness of respiratory viral identification among respiratory inpatients, Canada, 2003-2014. Influenza Other Respir Viruses 2018; 12: 113-121.

56 Chowell G, Bertozzi SM, Colchero MA, et al. Severe respiratory disease concurrent with the circulation of H1N1 influenza. N Engl J Med 2009; 361: 674-679.

57 Shiley KT, Nadolski G, Mickus T, et al. Differences in the epidemiological characteristics and clinical outcomes of pandemic (H1N1) 2009 influenza, compared with seasonal influenza. Infect Control Hosp Epidemiol 2010; 31: 676-682.

58 Guan W-J, Ni Z-Y, Hu Y, et al. Clinical characteristics of coronavirus disease 2019 in China. N Engl J Med 2020; 382: $1708-1720$.

59 Hancock K, Veguilla V, Lu X, et al. Cross-reactive antibody responses to the 2009 pandemic H1N1 influenza virus. N Engl J Med 2009; 361: 1945-1952. 